\title{
Erratum to: Transport across nanogaps using self-consistent boundary conditions
}

D. Biswas ${ }^{\mathrm{a}}$ and R. Kumar

Theoretical Physics Division, Bhabha Atomic Research Centre, 400085 Mumbai, India

Eur. Phys. J. B 85: 189 (2012)

Received 17 July 2012

Published online 3 September 2012 - (c) EDP Sciences, Società Italiana di Fisica, Springer-Verlag 2012

On page 4 , equation (23) should read

$$
\frac{d^{2} \bar{U}}{d \bar{x}^{2}}=q^{2} .
$$

In the line below equation $(24)$, "with $\bar{U}(0)=0, \bar{U}(1)=\bar{U}_{g}$." should $\operatorname{read}$ "with $\bar{U}=V / V_{s}, \bar{U}(0)=0$ and $\bar{U}(1)=\bar{U}{ }_{g} . "$.

\footnotetext{
${ }^{a}$ e-mail: dbiswas@barc.gov.in
} 